\title{
Contribution of the Refractive Index Fluctuations to the Length Noise in Displacement Interferometry
}

\author{
Miroslava Holá, Jan Hrabina, Martin Sarbort, Jindrich Oulehla, Ondrej Cíp, Josef Lazar \\ Institute of Scientific Instruments, Academy of Sciences of the Czech Republic, Královopolská 147, \\ 61264 Brno, Czech Republic
}

\begin{abstract}
We report on investigations of how fast changes of the refractive index influence the uncertainty of interferometric displacement measurements. Measurement of position within a limited range is typical for precise positioning of coordinate measuring systems, such as nanometrology standards combined with scanning probe microscopy (SPM). The varying refractive index of air contributes significantly to the overall uncertainty; it plays a role especially in case of longer-range systems. In our experiments we have observed that its fast variations, seen as length noise, are not linearly proportional to the measuring beam path and play a significant role only over distances longer than $50 \mathrm{~mm}$. Thus, we found that over longer distances the length noise rises proportionally. The measurements were performed under conditions typical for metrology SPM systems.
\end{abstract}

Keywords: Nanometrology, interferometry, refractive index of air.

\section{INTRODUCTION}

$\mathrm{D}$ IMENSIONAL METROLOGY on the macroscale as well as the nanoscale is a domain of interferometric techniques.

The concept of instrumentation for nanometrology has resulted in the combination of a microscope with a measuring system capable of delivering the accuracy and precision needed on the nanoscale with traceability to the primary length standard. The standard configuration consists of a scanning probe microscope (SPM) and a coordinate multiaxis positioning system which moves the stage with a sample with interferometric measurement of displacement [1], [2].

The demand for resolution of the positioning on the nanometer or even sub-nanometer level led to the development of systems operating only within a limited scanning range. This so-called short-range metrological SPM employs a piezo-driven stage held on flexure joints with virtually no backlash, and with high sensitivity [3], [4]. Here, the positioning range is below $1 \mathrm{~mm}$. Recently, the need to investigate larger samples, such as wafers for semiconductor industry, motivated development of long-range metrological SPMs with range in the horizontal plane up to several $\mathrm{cm}$. Long-range nanopositioning stages represent a mechanical challenge requiring designs with sophisticated flexure lever systems [5-9] or high-quality guidance reducing the backlash [10], [11]. Monitoring and control of the position through multiaxis interferometry shares the same problems with micro coordinate measuring machines (CMMs). Uncertainty budget of displacement measurement over a limited range includes laser frequency stability, laser and electronic noise, geometrical errors, resolution and linearity of the subdivision of one interferometric fringe, and contribution of the varying refractive index of air when the system operates under atmospheric conditions.

Commercial interferometric systems rely on indirect evaluation of the refractive index of air with measurement of air temperature, pressure, and humidity. Data obtained from system evaluation are consequently used for calculation of the wavelength correction with the help of the empirical Edlen formula [12] which has been gradually improved [9], [13 - 15]. Updated formulas include also the influence of the content of carbon dioxide in air. This technique is able to follow drifts of the refractive index of air with sufficient precision. However, this technique relies on measurement of the parameters of atmosphere (especially temperature) in one, or only in a few isolated places which do not give a possibility to get the information about the distribution of the refractive index within the beam path. Moreover, due to limited response time of the sensors it is not possible to follow fast changes that appear as a length noise.

Previously we proposed an interferometer designed in a way to operate within a specified measuring range, which compensates or at least significantly reduces the refractive index changes caused by length noise. We have proven the concept in a regime of stabilization of wavelength [16]. Moreover, in our recent work [17], [18] we reported an improved design of the interferometric setup which was tested also in a regime of tracking refractometer with in-line monitoring of the refractive index capability. During these experiments we observed the nature of the fluctuating refractive index of air through the recorded length noise over a measuring range (beam path) between 10 and $20 \mathrm{~cm}$. Note that even under laboratory conditions when the experimental setup was covered with an enclosure, a limited air flow inside cannot be avoided. The enclosure helped to control temperature through water filled walls reducing thermal gradients.

Opening of the interferometric setup to air flow determines the nature of the refractive index induced length noise. The enclosure opening raises not only amplitude, but also the speed of the variations and, thus, broadens the bandwidth of the noise towards higher frequencies. Slow changes of the refractive index of air could be interpreted as drift mostly due to temperature drift. This cannot be fully separated from the drift caused by thermal expansion of the mechanics of the positioning stage. Here we focused mainly on the question whether this length noise is simply proportional to the air beam path (measuring range of the interferometer when the dead length is minimized). If so, it could be seen as a random multiplicative contribution to the overall uncertainty. From the previous experiments we expected some kind of non-linear nature of the noise with a very limited contribution below some specific volume of air (beam path in air). 


\section{RANDOM NATURE OF THE PROPERTIES OF ATMOSPHERE}

When a laser beam propagates through the atmosphere, its properties are affected. The air cannot be considered as a homogeneous environment - it is a mixture of various gasses and there is always a transfer, or exchange of heat takes place. This results in a motion of air cells producing a turbulent behavior. From the optical point of view this means inhomogeneous and dynamically changing refractive index. The optical properties and dynamic behavior of the atmosphere have been studied widely on the large scale, primarily for applications in free-space optical (FSO) communications [19], Here, the communication distances and beam diameters are several orders larger compared to the dimensions considered in interferometry. The key problem in FSO communications is an effect called scintillations which is also a product of the varying refractive index of air. They produce random temporal and spatial irradiance fluctuations of the beam. Consequently, inhomogeneous refractive index over the beam path produces varying focusing and defocusing effects at the receiver [20], [21].

In case of interferometry, in the small scale, we can consider the scintillation effects negligible due to the diameter of the laser beam which we expect to be smaller compared to the size of the air cells involved in the turbulent behavior of air. The key process that influences the measurement uncertainty is the varying integral value of the refractive index over the beam path which produces variations of the optical length. In open-air conditions, under presence of an air flow, the velocity field in a turbulent state splits into a set of eddies of various sizes exchanging energy with one another. This process can be scaled to fit into a range limited by the largest and smallest turbulent eddy [22], where on the bottom of the scale the energy transfer occurring among the eddies ends up as dissipation of heat through viscosity. The scale can be expressed by sizes (lengths $l$ ) ranging between $L_{0}$ and $l_{0}$, where $L_{0}>l>l_{0}$. The length $l$ represents the length of an elementary air cell involved in the turbulent behavior of air which fits into a range given by the maximum and minimum size $\left(L_{0}\right.$ and $\left.l_{0}\right)$.

Random behavior of the parameters of atmosphere seen through the variations of the refractive index can be expressed as a random field in time and space and the refractive index at one specific point as a random variable in time. The index of refraction fluctuations caused by inhomogeneous and time-changing properties of the atmosphere can be expressed as:

$$
n(r, t)=n_{0}+n_{1}(r, t)
$$

where $r$ represents spatial position of the point of interest and $t$ the time variable. $n_{0}$ is the mean value of the index of refraction within the considered volume and over the time of interest and $\mathrm{n}_{1}(r, t)$ represents the random deviation from the mean value at the point $r$ and moment $t$. In our case, when we look at the laser beam in interferometry measuring the mechanical length being randomly affected by the varying optical length, the 3D position vector $r$ can be reduced to $2 \mathrm{D}$ because we evaluate the refractive index as one integral value over the whole beam path. With a fixed position of $r$ we concentrate on the time evolution of the random process over time. In this contribution we investigate the bottom end of the scale $l_{0}$ mentioned above and its influence on the varying optical length measured by a one-dimensional interferometer depending on the length of the beam path.

\section{EXPERIMENTAL CONFIGURATION}

Our experimental arrangement is shown in Fig.1. We combined a Michelson interferometer with the reference arm placed in an evacuated cell. The length of the air path varied from nearly zero up to several centimeters. The beam diameter was $3.5 \mathrm{~mm}$. Length of the reference arm was set to the middle of the translation range to reduce the influence of the laser frequency noise. The evacuated reference cell limited the sensitivity to the air flow only into the measuring arm. The plane-mirror configuration with an identical beam path in both directions was used.

The interferometer operates in a simple fringe-counting regime with $\lambda / 2$ resolution. Detection principle is homodyne with optical introduction of the $\lambda / 4$ delay. Quadrature analog signals are digitized (12-bit analog-to-digital conversion) and processed in a digital signal processor (DSP) unit with fringe counting embedded in the hardware and actual phase is calculated in a DSP processor. Hardware as well as software routines have been designed and developed at our institute. After the evaluation of the interference phase, the wavelength resolution of $\lambda / 2048$ results in a resolution of $260 \mathrm{pm}$ for $532 \mathrm{~nm}$ wavelength. The linearization technique implemented here reduces the linearity error to the level of a single discrete LSB (least significant bit) - the resolution of the interferometer [23], [24]. The operating wavelength is $\lambda$ $=532 \mathrm{~nm}$, and the laser source is a high-stability and lownoise, metrology grade frequency-doubled Nd:YAG laser. The laser was designed for multiaxis measuring systems for nanometrology and its stability ranges between $10^{-8}$ and $10^{-9}$ level. Together with stability recording it is described in more detail in [25].

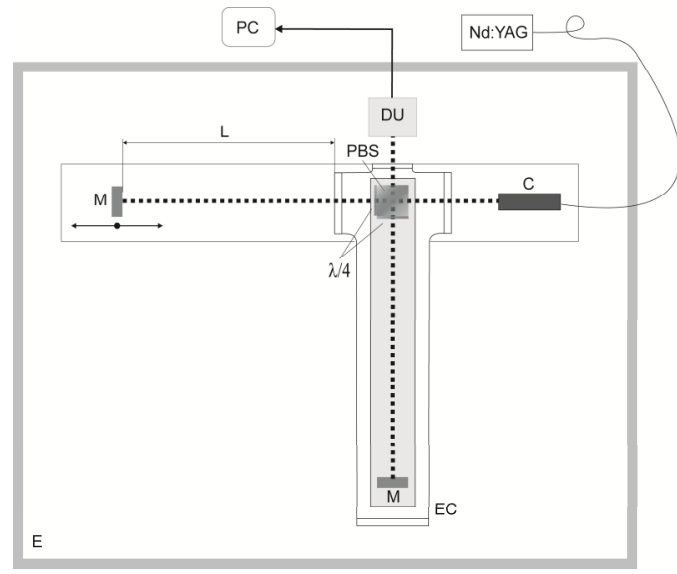

Fig.1. Interferometer setup for monitoring of the refractive index induced length noise with the reference arm placed in an evacuated cell. C: fiber collimator, DU: detection unit, E: enclosure, EC: evacuated cell, L: beam path on air, M: mirror, PBS: polarizing beam splitter, $\lambda / 4$ : retardation plate. 
The setup was placed in an enclosure with the intention to emulate operating conditions of most coordinate positioning systems for small-range CMMs and metrological long-range SPMs. The volume of the enclosure surely plays a role determining the level of air flow. As shown in [26], covering the laser beams in tubing reduces the length noise. We used a box with water filled walls reducing thermal gradients of the air inside. Internal air volume was 40 liters. The arrangement was placed on an antivibration table in an underground laboratory eliminating the influence of acoustic interference. Light delivery was fiber optic (polarization maintaining single-mode fiber) which allowed keeping the laser - representing a significant heat source - safely away from the experiment.

\section{EXPERIMENTAL RESULTS}

We have performed a series of measurements in order to investigate the amplitude of the refractive index induced length noise to the output from the length measuring/monitoring interferometer. The plane mirror was gradually moved over $2 \mathrm{~mm}$ from nearly zero air path up to $80 \mathrm{~mm}$ distance. At each position we recorded $20 \mathrm{~min}$ data sets with 100 samples per second acquisition rate and evaluated the root mean square (RMS) value of the length noise.

With the mirror in the measuring arm fixed during each recording there is a contribution of several sources of drift/noise. We focused on the refractive index induced noise by limiting the frequency bandwidth. Thus, all slow, long-term drifts with frequencies below $5 \mathrm{mHz}$ were removed from the recordings. They combine contributions from refractive index drift (primarily due to temperature) and mechanical thermal expansion. Dilatation of the metrology frame is a question of the design and proper thermal control and slow drift of the refractive index can be compensated the traditional way through the Edlen formula with acceptable precision. Upper limit of the frequency bandwidth of interest was $1 \mathrm{~Hz}$. Due to the low-noise laser and a good isolation of the setup from acoustic interference, over this frequency limit only the quantization noise of the analog-to-digital conversion dominates. In Fig.2. there is an example of one recording of the length noise with short $(10 \mathrm{~mm})$ beam path in the air. Fig.3. shows longer path $(80 \mathrm{~mm})$ with selected frequency bandwidth ranging from $5 \mathrm{mHz}$ up to $1 \mathrm{~Hz}$.

In Fig.2. and Fig.3. the original recordings including the quantization noise of the A/D conversion are marked black and the low-pass filtered signals used for calculation of the RMS value are marked red.

The resulting RMS value of the length noise with respect to the length of the measuring beam in air is shown at Fig.4. It is clearly visible that the dependency is not linear and thus the air does not behave proportionally the same way within different distance. Within the measuring range below $50 \mathrm{~mm}$ the fluctuations of length due to variations of the refractive index keep low and constant. In fact they can be considered as negligible. It is clearly visible that above $50 \mathrm{~mm}$ the noise starts to rise and is approximately proportional with the monitored distance (red line).

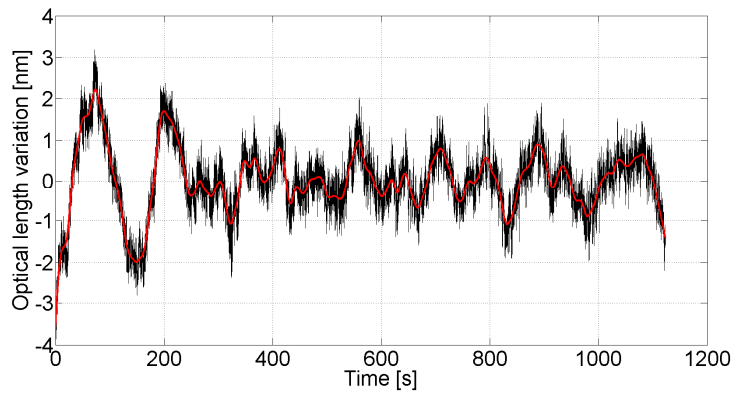

Fig.2. Recording of the length noise within $10 \mathrm{~mm}$ beam path on air, Black line: original recording, red line: quantization noise filtered away.

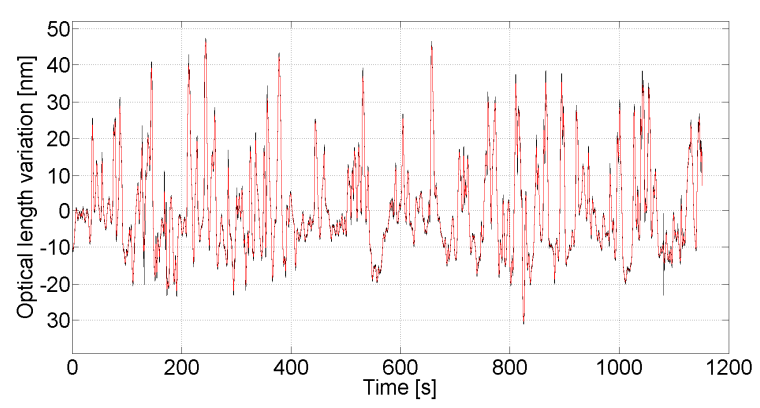

Fig.3. Recording of the length noise within $80 \mathrm{~mm}$ beam path on air, Black line: original recording, red line: quantization noise filtered away.

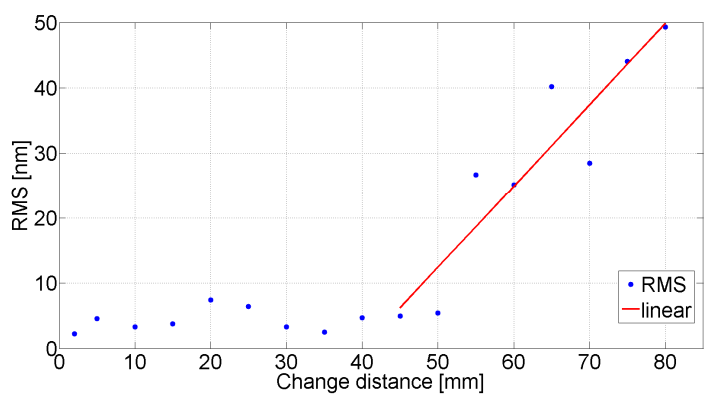

Fig.4. The resulting RMS values of the length noise with respect to the length of the measuring beam on air. Red line: approximated linear rise of the noise with the measured distance.

From the above mentioned findings we can conclude that the fast variations of the refractive index of air occur within some limited elementary volumes of air. The behavior of air even in an enclosed and steady environment shows a certain level of spontaneous air mixing/flow that cannot be completely avoided. Its influence on the noise of the measured value - displacement and overall uncertainty of the positioning and measuring system - is significant and has to be considered.

This investigation was motivated by application of highresolution interferometric techniques into multiaxis measuring systems in nanometrology and micro CMMs. This means measurement within a limited length range and an effort to reduce all sources of uncertainty. The configuration of the setup follows the conditions of these systems (laboratory conditions, enclosure, measuring range, 
beam diameter, etc.). The question we wanted to answer is whether it is useful to introduce relatively complex techniques of active compensation of the fluctuations of the refractive index of air we proposed before [17], [18]. The observed threshold length of about $50 \mathrm{~mm}$ refers under these conditions to the value $l_{0}$ of the air cells. It seems that below this threshold the compensation technique would not bring a useful improvement.

\section{DISCUSSION / CONCLUSIONS}

In this paper we have shown that when performing interferometric displacement measurements there is a limiting length (measuring range, beam path on air). When operating below this limit the length noise associated with refractive index of air is very small and is not linearly proportional to the measured displacement. On the contrary, when measuring above this limit the noise starts to rise linearly with the measured value. Note that there are relatively fast changes, not the slow drift. Absolute (mean) value of the refractive index of air can be evaluated the traditional way through the Edlen formula and contributes directly and linearly to the uncertainty. With the help of precise sensors to measure the fundamental parameters of atmosphere and new, improved versions of the formula, this value can be set quite precisely.

The case we presented here is a measurement with a single beam, one axis measurement. Differential measurement with two, or four parallel beams would make things different, depending on the beam spacing. Correlation of the length noise observed by parallel beams was presented in [27] and shows that closely spaced beams in differential configuration could produce a compensative effect.

Our observation can be exploited using the design of medium and small-range CMMs and metrological medium or long-range SPMs. When properly covered and shielded from the outer air flow and interference systems with range below our limit, the system can perform well with refractive index evaluated indirectly through the Edlen formula. In case of longer-range systems the refractive index noise contribution in the specific bandwidth has to be considered. To suppress these fast fluctuations it is necessary to measure the refractive index of air in real time directly in the beam path and to use it for compensation of the measured displacement, again in real time. With the bandwidth of this length noise below $1 \mathrm{~Hz}$, the demand for speed of processing is not high.

One of the concepts that could solve the problem of longer range positioning was proposed in [17], [18]. The best combination could be precise measurement of the parameters of the atmosphere, calculation of the wavelength correction through the Edlen formula following the slow drift and compensation of the faster length noise by the technique of in-beam tracking refractometry or active stabilization of the wavelength on air through servo-control of the laser optical frequency.

\section{ACKNOWLEDGEMENT}

The authors wish to express thanks for support to the grant projects from the Grant Agency of CR, project GB14$36681 \mathrm{G}$, the EMRP project IND58 6DoF which is jointly funded by the EMRP participating countries within EURAMET and the European Union and Technology Agency of CR, projects: TA02010711, TE01020233. The infrastructure for the research was funded by Ministry of Education, Youth and Sports CR, projects LO1212, and by Academy of Sciences CR, project RVO: 68081731. This project is co-financed by European social fund and state budget of CR, project No. CZ.1.07/2.3.00/30.0054

\section{REFERENCES}

[1] Leach, R.K., Boyd, R., Burke, T., Danzebrink, H.U., Dirscherl, K., Dziomba, T., et al. (2011). The European nanometrology landscape. Nanotechnology, $22(6)$.

[2] Neuschaefer-Rube, U., Neugebauer, M., Dziomba, T., Danzebrink, H.U., Koenders, L., Bosse, H. (2011). Recent developments of standards for 3D micro- and nanometrology. tm-Technisches Messen, 78 (3), 118126.

[3] Korpelainen, V., Seppa, J., Lassila, A. (2010). Design and characterization of MIKES metrological atomic force microscope. Precision Engineering, 34 (4), 735744.

[4] Haycocks, J., Jackson, K. (2005). Traceable calibration of transfer standards for scanning probe microscopy. Precision Engineering, 29 (2), 168-175.

[5] Jansen, A., Rosielle, N., Schellekens, P. (1999). A fully elastically guided 3-D CMM with a measuring volume or $1 \mathrm{~cm}(3)$. In Fourteenth Annual Meeting of the American Society for Precision Engineering. ASPE, 452-455.

[6] Poyet, B., Ducourtieux, S. (2010). Advances in the development of the LNE metrological atomic force microscope. In Optical Micro- and Nanometrology III. Proc. SPIE 7718.

[7] Werner, C., Rosielle, P.C.J.N., Steinbuch, M. (2010). Design of a long stroke translation stage for AFM. International Journal of Machine Tools \& Manufacture, 50 (2), 183-190.

[8] Von Seggelen, J.K., Rosielle, P.C.J.N., Schellekens, P.H.J., Spaan, H.A.M., Bergmans, R.H., Kotte, G.J.W.L. (2005). An elastically guided machine axis with nanometer repeatability. CIRP Annals Manufacturing Technology, 54 (1), 487-490.

[9] Haitjema, H., Rosielle, N., Kotte, G., Steijaert, H. (1998). Design and calibration of a parallel-moving displacement generator for nano-metrology. Measurement Science \& Technology, 9 (7), 10981104.

[10] Jager, G., Manske, E., Hausotte, T. (2006). New applications of the Nanomeasuring Machine (NPMMachine) by novel optical and tactile probes with subnanometer repeatability. tm-Technisches Messen, 73 (9), 457-464. 
[11] Eves, B.J. (2009). Design of a large measurementvolume metrological atomic force microscope (AFM). Measurement Science \& Technology, 20 (8).

[12] Edlen, B. (1966) The refractive index of air. Metrologia, 2 (2), 71-80.

[13] Birch, K.P., Downs, M.J. (1993). An updated edlen equation for the refractive-index of air. Metrologia, 30 (3), 155-162.

[14] Ciddor, P.E. (1996). Refractive index of air: New equations for the visible and near infrared. Applied Optics, 35 (9), 1566-1573.

[15] Birch, K.P., Downs, M.J. (1994). Correction to the updated edlen equation for the refractive-index of air. Metrologia, 31 (4), 315-316.

[16] Lazar, J., Cip, O., Cizek, M., Hrabina, J., Buchta, Z. (2011). Suppression of air refractive index variations in high-resolution interferometry. Sensors, 11 (8), 7644-7655.

[17] Lazar, J., Hola, M., Cip, O., Cizek, M., Hrabina, J., Buchta, Z. (2012). Refractive index compensation in over-determined interferometric systems. Sensors, 12 (10), 14084-14094.

[18] Lazar, J., Hola, M., Cip, O., Cizek, M., Hrabina, J., Buchta, Z. (2012). Displacement interferometry with stabilization of wavelength in air. Optics Express, 20 (25), 27830-27837.

[19] Mujumdar, A.K. (2014). Advanced Free Space Optics (FSO). Springer.
[20] Andrews, L.C., Phillips, R.L., Hopen, C.Y. (2001) Laser Beam Scintillation with Application. SPIE.

[21] Ricklin, J.C., Hammel, S.M., Eaton, F.D., Lachinova, S.L. (2008). Atmospheric channel effects on freespace laser communication. In Free-Space Laser Communications: Principles and Advances. Springer, 9-56.

[22] Wheelon, A.D. (2001). Electron Scintillation. Cambridge University Press.

[23] Cip, O., Petru, F. (2000). A scale-linearization method for precise laser interferometry. Measurement Science \& Technology, 11 (2), 133-141.

[24] Petru, F., Cip, O. (1999) Problems regarding linearity of data of a laser interferometer with a singlefrequency laser. Precision Engineering, 23, 39-50.

[25] Hrabina, J., Lazar, J., Klapetek, P., Cip, O. (2011). Multidimensional interferometric tool for the local probe microscopy nanometrology. Measurement Science \& Technology, 22 (9).

[26] Ducourtieux, S., Poyet, B. (2011). Development of a metrological atomic force microscope with minimized Abbe error and differential interferometer-based realtime position control. Measurement Science \& Technology, 22 (9).

[27] Hola, M., Lazar, J., Cip, O., Buchta, Z. (2014). Inbeam tracking refractometry for coordinate interferometric measurement. In Optical Micro- and Nanometrology V. Proc. SPIE 9132. 